\title{
Incidental finding of von Meyenburg complexes mimicking liver metastases on routine laparoscopic cholecystectomy
}

\author{
Shaveen D Kanakaratne ${ }^{1}$, Gayatri Asokan ${ }^{2}$, Chandika Liyanage ${ }^{1}$ \\ ${ }^{1}$ Royal Prince Alfred Hospital, Camperdown, New South Wales, Australia \\ ${ }^{2}$ Royal Darwin Hospital, 105 Rocklands Drive, Tiwi, Northern Territory, Australia
}

Keywords: Hepatobiliary surgery; biliary hamartomas

\section{Introduction}

First described in 1918, Von Meyenburg complexes (VMC) or bile duct hamartomas, are a rare benign epithelial tumour of liver derived from bile duct cell $[1,2]$. They are mostly asymptomatic and found incidentally. Their differential diagnoses include Caroli disease, parasitic liver cysts and rarely metastatic disease [3].

A few case reports were found with patients presenting with nonspecific abdominal pain or as an infective complications of liver parenchyma [3]. This case report outlines an incidental intraoperative finding of von Meyenburg complexes and subsequent management in a 57 year old male which mimicked liver metastases on routine laparoscopic cholecystectomy.

\section{Case presentation}

A 57 year old, previously well Caucasian male was planned to have a laparoscopic cholecystectomy following ERCP for choledocholithiasis. A diagnostic laparoscopy and biopsy was performed instead, in view of intraoperative findings of multiple $5 \mathrm{~mm}$ to $15 \mathrm{~mm}$ nodular white deposits widespread over liver (figure 1). Concerning for liver metastases, biopsies were taken from Segment IVB of liver as frozen section histopathology facilities were unavailable. The histopathology returned as benign bile duct hamartomas (VMC, von Meyenburg complexes). The microscopic description read "small clusters of benign bile duct glands, glands are variably angulated and lined by cuboidal cells containing inspissated bile within lumen. Glands are surrounded by fibrous stroma. Mild incidental hepatic steatosis".

Given benign nature, a laparoscopic cholecystectomy was started although converted to an open subtotal cholecystectomy due to an abnormal duct from the liver entering the

Correspondence: Shaveen D Kanakaratne

E-mail: skanakaratne@yahoo.com.au

Received: 13-01-2018 Accepted: 27-03-2018

(iD https://orcid.org/0000-0001-7895-2122

DOI: http://doi.org10.4038/sljs.v36i1.8486

The Sri Lanka Journal of Surgery 2018; 36(1): 49-50

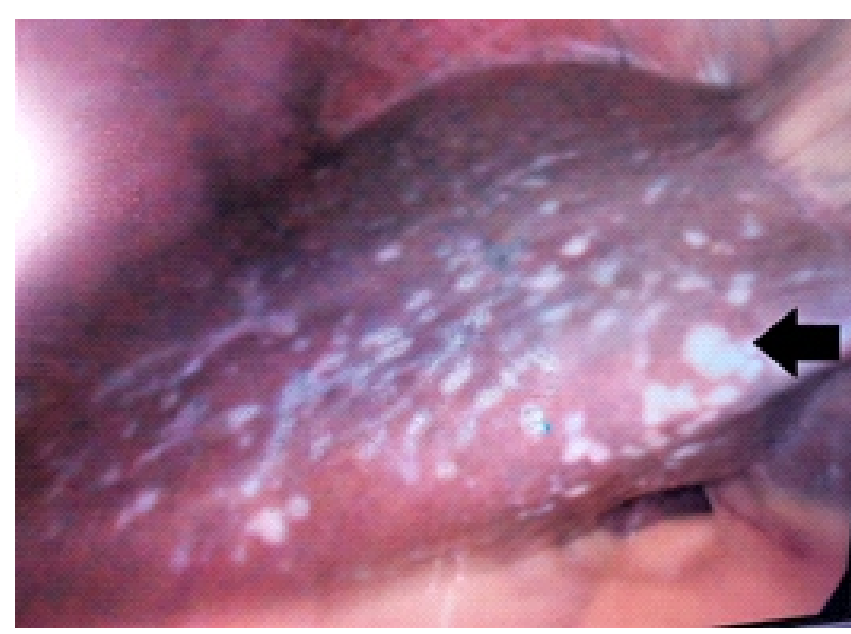

Figure 1. Intraoperative Image: Segment 4B liver anterior surface demonstrating VMC complexes

distal Hartmann's pouch, close to his cystic duct. The histopathology from the subtotal cholecystectomy returned as active chronic cholecystitis. He was discharged from hospital with follow up made with tertiary centre gastroenterology and hepatobiliary teams.

\section{Conclusion}

VMC's are a rare benign epithelial tumor of liver derived from bile duct cell $[1,2]$. Macroscopically they are described as multiple small nodular lesions, with a size less than $1.5 \mathrm{~cm}$ and having a cystic appearance. They are thought to be caused by small intrahepatic bile ducts undergoing ductal plate malformation, secondary to disordered embryonic involution $[1,2$, 3]. They can be classed as solid, intermediate or cystic [4].

Diagnostic criteria require histology showing cystic dilation of bile ducts embedded in fibrous stroma with no evidence of malignancy. The differential diagnosis of liver cysts includes Caroli disease, parasitic liver cysts and rarely metastatic disease [3].

Mostly asymptomatic, a few cases report patients presenting with nonspecific abdominal pain or as an infective complications of liver parenchyma and cholangitis [3].

Imaging modalities may identify VMC and they include 
ultrasound with features of numerous hyperechoic or hypoechoic areas with "comet tail echoes" [3]. CT scan may show small hypodense nodules with ring like enhancements [3]. Magnetic Resonance Imaging (MRI) is preferred as it can distinguish saccular dilation of Caroli disease versus peri ductal cystic dilation of polycystic disease [3]. None of above were seen on our patient's ultrasound and CT scan. Literature suggests that in CT or MRI scanning, the lack of enhancement post intravenous contrast and lack of communication with biliary tree, helps distinguish bile duct hamartomas from malignancy $[3,5]$.

Bile duct hamartomas may coincide with any liver tumor benign or malignant [2]. This necessitates liver biopsies if the diagnosis is in doubt $[2,4]$. Incidental finding in laparoscopy and autopsy is 0.7 to 2.8 percent prevalence [3]. In all literature reviewed, frozen section or biopsy were performed by the surgeons to confirm the diagnosis and exclude hepatic metastases.

In conclusion, literature search and review indicate that Von Meyenburg Complexes can be encountered incidentally by surgeons as undifferentiated liver nodules intraoperatively and hence biopsies should be performed to exclude other differentials.
All authors disclose no conflict of interest. The study was conducted in accordance with the ethical standards of the relevant institutional or national ethics committee and the Helsinki Declaration of 1975, as revised in 2000 .

\section{References}

1. Lorenzon L, Ziparo V. Von Meyenburg complexes mimicking liver metastases. Hepatology. 2011 Aug; 54(2):734-5. https://doi.org/10.1002/hep.24220

2. Bieze, $M$ et al. Von Meyenburg complexes mimicking metastatic disease at laparotomy for focal nodular hyperplasia. BMJ Case Rep. 2013 Jun 26; 2013. https://doi.org/10.1136/bcr-2013010250

3. Sinakos, E et al. The clinical presentation of Von Meyenburg complexes. Hippokratia. 2011, 15, 2: 170-173. Accessed from (http://www.hippokratia.gr/images/PDF/15-2/749.pdf)

4. Ioannidis, $\mathrm{O}$ et al. Incidentally discovered white subcapsular liver nodules during laparoscopic surgery: biliary hamartoma and peri biliary gland hamartoma. Klin Onkol. 2012;25(6):468-70. Article accessed from http://www.prolekare.cz/pdf?id=39495.

5. Davidoff S, Kim S, Friedman B. Von Meyenburg complexes (bile duct hamartomas). Clinical Gastroenterology and Hepatology. 2006; 4: xxvi. https://doi.org/10.1016/j.cgh.2005.10.010

\section{Learning Points:}

- Undifferentiated liver nodules can be encountered by surgeons intraoperatively.

- When encountered in an intraoperative setting, undifferentiated liver nodules should be biopsied.

- Von Meyenburg complexes (VMC) or bile duct hamartomas are a rare benign epithelial tumor of the liver, although can still be encountered in an intraoperative setting, biopsies should be performed to differentiate from more sinister pathology. 